\title{
THE PROBABILISTIC BROSAMLER FORMULA FOR SOME NONLINEAR NEUMANN BOUNDARY VALUE PROBLEMS GOVERNED BY ELLIPTIC POSSIBLY DEGENERATE OPERATORS
}

\section{GREGORIO DÍAZ}

Abstract. This paper concerns with boundary value problems as

$$
\begin{cases}\mathscr{L} u+a_{0} u=f & \text { in } \Omega, \\ <\nabla u, \vec{\gamma}>+c_{0}|u|^{\mathrm{m}-1} u=g & \text { on } \partial \Omega,\end{cases}
$$

where $\mathscr{L}$ is an elliptic possibly degenerate second order operator, $a_{0}, c_{0}$ are positive function, $\vec{\gamma}$ is an oblique exterior vector and $\mathrm{m} \geqslant 1$. By means of some arguments close to the Dynamics Programming we prove that the viscosity solution admits a representation formula that can be considered as an extension of probabilistic Brosamler formula of linear Neumann boundary value problems governed by uniformly elliptic operators. Although other generalizations are possible, by simplicity we limit this contribution to the presence of nonlinear terms exclusively on the boundary of the domain. We emphasize that any uniforme ellipticity assumption is required in the paper.

Mathematics subject classification (2010): 35D40, 35J65, 60J60.

Keywords and phrases: non linear Neumann boundary condition, viscosity solutions, probabilistic approach, reflection diffusion.

\section{REFERENCES}

[1] G. BARLES, Nonlinear Neumann boundary conditions for quasilinear degenerate elliptic equations and applications, J. Differential Equations, 154 (1999), 191-224.

[2] G. BARLeS AND J. BuRdeAU, The Dirichlet problem for semilinear second-order degenerate elliptic equations and applications to Stochastic Control, Comm. in P.D.E., 20, (1\&2) (1995), 129-178.

[3] G. BARLeS et P. L. Lions, Remarques sur les problèmes de réflexion oblique, C. R. Acad. Sc. Paris, 320, Serie I (1995), 69-74.

[4] G. BARLes AND E. Roury, Deterministic and stochastic exit time control problems and HamiltonJacobi-Bellman equations with generalized Dirichlet boundary problems, to appear.

[5] A. BenchÉRIF-MADANI ANd É. PARdoux, Probabilistic formula for a Poisson equation with Neumann boundary condition, Stochastic Analysis and Applications, 27 (2009), 739-746.

[6] G. A. Bros AmLER, A probabilistic solution of the Neumann problem, Math. Scand., 38 (1976), $137-$ 147.

[7] M. G. Crandall, H. Ishii and P. L. Lions, User's guide to viscosity solutions of second order partial differential equations, Bull. Amer. Math. Soc., 27 (1992), 1-42.

[8] G. DíAZ, On the Dirichlet boundary problem for quasi-degenerate elliptic linear equations, in Contribuciones matemáticas, Homenaje al profesor Enrique Outerelo Domínguez, Universidad Complutense de Madrid, (2004), 103-126.

[9] W. Fleming And H. Mete Soner, Controlled Markov Processes and Viscosity Solutions, SpringerVerlag, 1993.

[10] M. I. Freidlin, Functional Integration and Partial Differential Equations, Annals of Mathematics Studies, 109, Princeton University Press, 1985. 
[11] D. Gilbarg And N. S. Trudinger, Elliptic Partial Differential Equations of Second Order, Springer-Verlag, 1983.

[12] P. Hsu, Probabilistic approach to the Neumann problem, Commun. Pure Appl. Math., 38 (1985), 445-472.

[13] O. LEY, A counter-example to the characterization of the discontinuous value function of control problems with reflections, C. R. Acad. Sci. Paris, Ser. I 335 (2002), 469-473.

[14] P. L. Lions, Control of diffusion processes in $\mathbb{R}^{N}$, Comm. Pure and Appl. Math., 34 (1981), 121-147.

[15] P. L. LiONS, Quelques remarques sur les problèmes elliptiques quasilineares de second ordre, Journal d'Analyse Mathematique, Vol 24 (1985), 234-254.

[16] P. L. Lions AND A. S. SZnitman, Stochastic differential equations with reflecting boundary conditions, Commun. Pure Appl. Math., 37, (4) (1984), 511-537.

[17] P. L. Lions AND N. S. TRUdinger, Linear oblique derivative problems for the uniformly elliptic Hamilton-Jacobi-Bellman equation, Math. Z., 191 (1986), 1-15.

[18] I. Karatzas And S. E. Shreve, Brownian Motion and Stochastic Calculus, Springer, 1988.

[19] P. MARÍN AND J. REAL, Some results on stochastic differential equations with reflecting boundary nonditions, J. Theoret. Probab., 17, 3 (2004), 705-716.

[20] V. G. PapanicolaOU, The probabilistic solution of the third boundary value problem for second order elliptic equations, Probab. Theory and Related Fields, 87 (1990), 27-77.

[21] Y. SAISHO, Stochastic differential equations for multi-dimensional domain with reflecting boundary conditions, Probab. Theory and Related Fields, 74 (1987), 455-477.

[22] J. Simon, Regularité de la solution d'une équation non linéaire dans $\mathbb{R}^{\mathrm{N}}$, In Lecture Notes in Mathematics, 665, Ph. Bénilan and J. Roberts (eds.), Springer (1987), 205-227.

[23] R. Situ, Reflecting Stochastic Differential Equations with Jump and Applications, Chapman \& Hall/CRC Press. 2000.

[24] A. Soriano, On some elliptic Dynamic Programming equations: Dirichlet conditions, Adv. Math. Sci. Appl., 9, No. 2 (1999), 805-816.

[25] J. Yong AND X. T. Zhou, Stochastic Control, Springer-Verlag, 1999. 